\title{
Classification of ECG Signals By the Neighborhood Feature Extraction Method
}

\author{
C.Bakir
}

\begin{abstract}
In this study, non-linear dimension reduction methods were applied to ECG signals and success of such dimension reduction techniques for the classification and segmentation of ECG signals were discussed. Also, segmentation of data through neighbourhood feature extraction (NFE) method were enabled by transiting from high dimensioned space to low dimension space by considering the longitudinal combination of ECG signals. Results classification results of NFE algorithm performed through longitudinal combination and as a newly developed method were compared with classification results of ECG signals obtained through dimension reduction by taking one pixel. Results of NFE dimension reduction technique performed by considering the neighbour pixels, advantage of effect on segmentation of ECG signals were presented at empirical results section and the success of suggested method was indicated. Results obtained by performed study are promising for the studies to be conducted in further period.
\end{abstract}

Index Terms-classification; ECG; kNN; Neighbourhood Feature Extraction; RBF.

\section{INTRODUCTION}

$\mathrm{E}$ LECTROCARDIOGRAM (ECG) means electrical signs, which show how the heart works in the human body and also show heart motions. Cardiac diseases arisen depending on cardiac arrhythmias, and cardiologic disorders and anomalies occurred in heart are diagnosed by ECG signals. Therefore, it is very important today that ECG signals can be analyzed properly [1].

Today most people die depending on cardiac diseases. Therefore, attention must be paid to heart health. ECG records any changes in the electrical signs generated in the heart and dispersing to all body and tries to diagnose any disorders occurred in the heart. ECG shows briefly the heartbeat and is used commonly in signal processing [2].

Most studies are conducted on classification of ECG signals. But, in the studies, it is not ensured exactly that ECG signals may be understood quickly and interpreted properly. The new method developed together with this study is applied successfully to all dimension reduction techniques. Furthermore, success to classify any ECG signals is increased and it is endeavored to guide any studies to be conducted in other biomedical fields as well.

C. BAKIR, is with Department of Computer Engineering University of Yildiz Technical University, Istanbul, Turkey, (e-mail: cigdem@ce.yildiz.edu.tr).
In the study, firstly a stripe selection and feature extraction are conducted for dimension reduction. In this study, it is endeavored to conduct a classification by the instructional methods and by utilizing the feature extraction in ECG data and using the class labels. There are most methods to conduct the feature extraction. Of these methods, the oldest and most known linear technique is PCA [3]. But, the linear techniques are not sufficient to obtain any information. Any non-linear techniques are required to classify any complex data. To that end, most nonlinear techniques are used recently. In our study, the nonlinear techniques, which keep the local properties such as Kernel PCA, Isomap, etc., are used for our existing dataset as well as the non-linear feature extraction techniques, which keep the global properties such as such as Laplacian Eigenmaps, etc. Furthermore, the linear extraction techniques such as PCA and LDA are also used to compare the classification success with the non-linear techniques [4].

Secondly, we discuss $5,10,15,20$ and 50 extensional neighborhoods for our dataset generated by us by these feature extraction methods and try to classify ECG data [5]. In ECG data, each data is stated as a sample point. This example point is called "pixel." The vectors generated for these pixels are valid for all stripes and have a particular value. If class of a pixel is certain, class of the pixel immediately adjacent to that pixel is the same as that pixel and this adjacent pixel shows the extensional neighborhood. $n \times n$ neighbor around this extensional neighborhood is taken for ECG signal as ECG signal is trained. Proximity of the extensional neighborhood between two points is measured by Euclidean distance. Two close data points are similar in extensional neighborhoods and the likelihood that these points are in the same class is high. For $n \times n$ neighbor, similarity reduces as distance increases.

\section{METHOD}

A large amount of data is dealt with in the real world. Therefore, much more memories and calculations are required to classify data properly. Data is used efficiently as data reduction and cost is reduced. To conduct this transaction without any data loss, the dimension reduction methods are required to ensure the dimension reduction and to reduce number of stripes. The feature extraction constitutes a very important step in processing the high dimensional data. The feature extraction is conducted by the linear or nonlinear methods on the stripes. In this study, firstly the feature extraction is conducted to perform an instructional study, to increase the classification success and to reduce the transaction cost by using the class information. 
Signatures are taken that provide any particular properties primarily to process all data. These spectral signatures are identified as ones that represent the spectral distribution in the best way as much as possible. The techniques of nonlinear feature extraction are applied to these spectral signatures instead of all data. The existing dataset is obtained by the dimension reduction techniques used in the study by extracting 5- and 10-dimensional vectors. Furthermore, RBF and kNN interpolation is used to instruct all dataset. The dataset reduced by RBF is learnt by an artificial plexus and it is moved from each spectral signature from a low dimensional space by using a network structure. RBF is formed by linkages between neurons. At the education stage, weights of the neurons are updates by examining inputs and outputs. This transaction continues until the error rate will be minimum [6]. In $\mathrm{kNN}$ interpolation method, $\mathrm{k}$ is found depending on the nearest samples. The nearest neighborhood of the data normalized in $\mathrm{kNN}$ interpolation is found and weights are calculated [7]. In this method, the spectral signatures and distances of each pixel in EKG data are found. Then, the value of $\mathrm{k}=9$ is selected and the nonlinear dimension reduction methods of the spectral signature $\mathrm{k}$ nearest to the pixel with a dimension to be reduced are found and the feature vectors are obtained. Here, the purpose is to find the nearest intermediate assessment to be obtained by the nonlinear projection methods. This method is applied to all spectral signatures and the dimension is reduced.

\section{A. Principal Component Analysis (PCA)}

Principal Component Analysis (PCA) is most popular orthogonal linear transformation. PCA is an illustration of the data having the biggest variance in the low dimensional space. High variance features are prepared with respect to low variance. $\mathrm{M}$ linear mapping of samples of $\mathrm{X}$ data matrix is founded that increases the cost function, calculating the covariance matrix. It gives the eigenvectors corresponding to the biggest eigenvalue. PCA uses the Euclidean distance between the data points $x_{i}$ and $x_{j}$. PCA transformation is shown as $\mu^{\mathrm{T}}=\mathrm{X}^{\mathrm{T}} \mathrm{W}$. W shows the orthogonal matrix; $\mu^{\mathrm{T}}$ shows the linear transformation, and $\mathrm{W}$ shows the eigenvectors corresponding to the covariance matrix [8].

PCA is a linear illustration of the data. The data is separated by PCA as shown in Equation 1 [8].

$$
x_{i}=\sum_{j=1}^{p} w_{i j} Q_{j}
$$

\section{B. Linear Discriminant Analysis (LDA)}

PCA states a high variety of data by a minimum number of components. But, these components never require best variety to classify them differently to ensure highest separation. To classify the data properly together with LDA, highest separation between different classes of data is ensured. It finds the dimension reduction required to provide this separation, calculating the covariance matrix. Total covariance matrix is calculated, utilizing the covariance matrix within the classes $\left(\mathrm{S}_{\mathrm{W}}\right)$ and the covariance matrix between the classes $\left(\mathrm{S}_{\mathrm{B}}\right)[1]$.

$$
\mathrm{S}_{\mathrm{T}}=\mathrm{S}_{\mathrm{W}}+\mathrm{S}_{\mathrm{B}}
$$

\section{Kernel Principal Component Analysis (KPCA)}

KPCA is an expanded version of PCA method. But, KPCA is a nonlinear technique, which improves the linear techniques, while PCA is a linear method. Dimension of the data is reduced, using the Kernel matrix. The $\mathrm{K}$ kernel matrix is calculated, using the data points $x_{i}$ and $x_{j}$ as $k_{i j}=\mathrm{K}\left(x_{i}, x_{j}\right)$. Centers and $\mathrm{d}$ vectors are found, changing inputs of the $\mathrm{K}$ kernel matrix. Thus, the data is transferred by means of KPCA to a higher dimensional space according to PCA dimension reduction technique. In KPCA, the important thing is to select the kernel function. Kernel function may be a linear kernel, Gauss kernel and polynomial kernel. In the studies conducted in the literature, KPCA provides fairly successful results in face recognition and speech recognition $[9,10]$.

\section{Isomap}

Isomap is a low dimensional embedding method, which is used in multi-dimensional scaling algorithms on a weighted graphic and uses a geodesic distance instead of an Euclidean distance. The geodesic distance is the shortest distance and to find this distance, neighborhoods between all data points must be found. The geodesic distance data points are calculated by $x_{\mathrm{i}} x_{j}((\mathrm{i}=1,2, \ldots \ldots, \mathrm{n})$ in the neighbor graphic. Furthermore, it is also important to select a neighborhood parameter in Isomap. The linkage in each data point is known as the nearest Euclidean distance in a high dimensional space. The nearest distance between two points is calculated by the Dijkstra algorithm [11].

\section{E. Laplacian Eigenmaps}

It ensures that the data is transferred to a low dimensional space, keeping its manifold local properties. The Laplacian method firstly composes the $\mathrm{G}$ graphic in conjunction with its nearest neighborhood $k$ and selects a weight. For the data points $x_{i}$ and $x_{i}$, the edge weights are calculated by Gauss. In the cost function in illustration in a $y_{i}$ low dimensional space, the weights $w_{i}$ depend on short distances between the data points $x_{i}$ and $x_{j}$. Thus, $y_{i}$ and $y_{j}$ minimize the cost function by the spectral graphic theory. M degree matrix is stated as $\mathrm{W}$ diagonal matrix in L laplacian graphic. Self-separation of the Laplacian graphic is conducted and a low dimensional embedding is created [12].

\section{EXPERIMENTAL STUDY AND RESULTS}

In this study, PTB (Physikalish-Techniseche Bundesantalt) ECG dataset is used. This dataset is generated by taking 549 ECG signals by the German National Metrology Institute from 289 people. 9 different diagnoses are made on the patients depending on heart diseases. Furthermore, each entry contains 15 signals measured simultaneously. Each signal is digitalized approximately by 1000 samples per second [13]. 
TABLE I

RESULTS OF RBF CLASSIFICATION ALGORITHMS FOR DIMENSIONS 5 AND 10

\begin{tabular}{|c|c|c|}
\hline \multirow{2}{*}{$\begin{array}{l}\text { Feature } \\
\text { Extraction } \\
\text { Methods }\end{array}$} & \multicolumn{2}{|c|}{$R B F$} \\
\hline & 5 dimensions & 10 dimensions \\
\hline PCA & 72.65 & 73.84 \\
\hline LDA & 68.66 & 65.37 \\
\hline KPCA & 78.13 & 80.31 \\
\hline Isomap & 79.04 & 79.56 \\
\hline Laplacian E. & 80.25 & 82.03 \\
\hline
\end{tabular}

TABLE II

RESULTS OF kNN CLASSIFICATION ALGORITHMS FOR DIMENSIONS 5 AND 10

\begin{tabular}{|c||c|c||}
\hline \hline \multirow{2}{*}{$\begin{array}{c}\text { Feature } \\
\text { Extraction } \\
\text { Methods }\end{array}$} & \multicolumn{2}{|c|}{$k \boldsymbol{N}$} \\
\cline { 2 - 3 } & 5 dimensions & 10 dimensions \\
\hline \hline PCA & 75.83 & 75.25 \\
\hline \hline LDA & 65.72 & 69.84 \\
\hline \hline KPCA & 84.35 & 85.04 \\
\hline Isomap & 85.67 & 83.39 \\
\hline Laplacian E. & 86.69 & 88.34 \\
\hline
\end{tabular}

The results obtained by kNN interpolation for dimensions 5 and 10 are shown in Table 2, while the classification results obtained by RBF for dimensions 5 and 10 are shown in Table 1. In Table 3, the results of classification done by RBF in NFE technique and suggested by ensuring the extensional combination for dimension 5 are shown, and in Table 4, the results of classification done for dimension 10 are shown.

TABLE III

THE RBF CLASSIFICATION RESULTS OF THE DATA OBTAINED BY NFE TECHNIQUE FOR DIMENSIONS 5

\begin{tabular}{|c||c|c|c||c||c||}
\hline \multirow{2}{*}{$\begin{array}{c}\text { Feature } \\
\text { Extraction } \\
\text { Methods }\end{array}$} & \multicolumn{5}{|c||}{ Neighborhoods Values } \\
\cline { 2 - 6 } & $\mathbf{5}$ & $\mathbf{1 0}$ & $\mathbf{1 5}$ & $\mathbf{2 0}$ & $\mathbf{5 0}$ \\
\hline \hline PCA & 75.87 & 85.34 & 87.62 & 85.34 & 64.37 \\
\hline \hline LDA & 70.12 & 75.82 & 79.87 & 80.12 & 60.72 \\
\hline \hline KPCA & 82.88 & 90.12 & 92.11 & 87.64 & 70.37 \\
\hline \hline Isomap & 83.17 & 88.72 & 95.64 & 87.62 & 71.64 \\
\hline \hline Laplacian E. & 84.62 & 90.62 & 97.83 & 94.62 & 74.83 \\
\hline
\end{tabular}

TABLE IV

THE RBF CLASSIFICATION RESULTS OF THE DATA OBTAINED BY NFE TECHNIQUE FOR DIMENSIONS 10

\begin{tabular}{|c|c|c|c|c|c|}
\hline \multirow{2}{*}{$\begin{array}{c}\text { Feature } \\
\text { Extraction } \\
\text { Methods }\end{array}$} & \multicolumn{5}{|c|}{ Neighborhoods Values } \\
\hline & 5 & 10 & 15 & 20 & 50 \\
\hline PCA & 75.01 & 77.83 & 80.64 & 78.62 & 64.33 \\
\hline LDA & 71.64 & 79.65 & 74.62 & 73.13 & 60.06 \\
\hline KPCA & 85.37 & 87.83 & 91.62 & 85.12 & 73.62 \\
\hline Isomap & 82.64 & 89.67 & 91.24 & 83.64 & 77.37 \\
\hline Laplacian E. & 84.65 & 90.83 & 95.62 & 92.47 & 78.12 \\
\hline
\end{tabular}

TABLE V

THE kNN CLASSIFICATION RESULTS OF THE DATA OBTAINED BY NFE TECHNIQUE FOR DIMENSIONS 5

\begin{tabular}{|c||c||c|c|c|c||}
\hline \multirow{2}{*}{$\begin{array}{c}\text { Feature } \\
\text { Extraction } \\
\text { Methods }\end{array}$} & \multicolumn{5}{|c|}{ Neighborhoods Values } \\
\cline { 2 - 6 } & $\mathbf{5}$ & $\mathbf{1 0}$ & $\mathbf{1 5}$ & $\mathbf{2 0}$ & $\mathbf{5 0}$ \\
\hline \hline PCA & 80.62 & 87.64 & 89.63 & 82.52 & 70.03 \\
\hline \hline LDA & 70.62 & 73.52 & 75.64 & 73.27 & 64.87 \\
\hline \hline KPCA & 87.64 & 92.02 & 96.75 & 91.54 & 71.64 \\
\hline \hline Isomap & 89.64 & 93.52 & 98.64 & 97.62 & 78.54 \\
\hline \hline Laplacian E. & 89.12 & 95.52 & 99.14 & 96.82 & 71.87 \\
\hline \hline
\end{tabular}

In Table 5, the results of classification done by $\mathrm{kNN}$ interpolation in NFE technique and suggested by ensuring the extensional combination for dimension 5 are shown, and in Table 6, the results of classification done for dimension 10 are shown.

TABLE VI

THE kNN CLASSIFICATION RESULTS OF THE DATA OBTAINED BY NFE TECHNIQUE FOR DIMENSIONS 10

\begin{tabular}{|c|c|c|c|c|c|}
\hline \multirow{2}{*}{$\begin{array}{c}\text { Feature } \\
\text { Extraction } \\
\text { Methods }\end{array}$} & \multicolumn{5}{|c|}{ Neighborhoods Values } \\
\hline & 5 & 10 & 15 & 20 & 50 \\
\hline PCA & 78.62 & 83.64 & 85.72 & 84.17 & 65.67 \\
\hline LDA & 74.44 & 76.62 & 79.87 & 75.67 & 61.12 \\
\hline KPCA & 90.67 & 94.86 & 97.61 & 95.62 & 76.15 \\
\hline Isomap & 84.61 & 88.63 & 90.82 & 87.46 & 71.04 \\
\hline Laplacian E. & 90.26 & 95.65 & 98.79 & 87.54 & 78.22 \\
\hline
\end{tabular}

In the study conducted in Table 1 and Table 2, it is observed that the suggested method increases the classification success 
considerably based on the results suggested in Tables $3,4,5$ and 6. Especially the neighborhood value 15 has highest classification success. But, a considerable increase in neighborhood reduces the classification success.

It is observed that NFE technique increase the success in classification of ECG signals more than $10 \%$ in both classification algorithms.

\section{CONCLUSION}

In this study, non-linear dimension reduction methods were applied to ECG signals. Also, segmentation of data through neighbourhood feature extraction (NFE) method by transiting from high dimensioned space to low dimension space by considering the spatial combination of ECG signals with results classification of traditional nonlinear feature extraction were compared. In this study, an extensional combination is provided and advantages of the dimension reduction on classification are submitted. Success of NFE technique suggested in this study on classification of ECG signals is shown. In any studies to be done in the future, it shall be ensured that NFE technique shall be improved further and applied in different practice fields.

\section{REFERENCES}

[1] Kiranyaz S., Ince T. etc, "Personalized long-term ECG classification: A systematic approach", Expert Systems with Applications, vol.38, issue 4, pp.3220-3226, 2011.

[2] Erdoğmuş P., Peşçaker A., "Dalgacık Dönüşümü ile EKG Sinyallerinin Özellik Çıkarımı ve Yapay Sinir Ağları ile Sınıflandırılması", 5.Uluslararası İleri Teknolojiler Sempozyumu (IATS'09), 2009.

[3] Martis R., Acharya R. And Min L., "ECG beat classification using PCA, LDA, ICA and Discrete Wavelet Transform", Elseiver Biomedical Sifnal Processing and Control, vol.8, issue 5, pp.437-448, 2013.

[4] Ceylan R.,Ozbay Y., "Comparison of FCM, PCA and WT techniques for classification ECG arrhytmias using neural network, Expert Systems with Applications, vol.33, issue 2, pp.286-295, 2007.

[5] Bakir C., "Nonlinear Feature Extraction for Hyperspectral Images", International Conference on Advanced Technology \& Sciences (ICAT'14), pp.945-949, 2014.

[6] Silipo R., Bortolan G. And Marchesi C., "Design of hybrid architectures based on neural RBF pre-processing for ECG analysis", International Journal of Approximate Reasoning, pp.177-196, 1999.

[7] Castillo O., Melin P. ETC, "Hybrid intelligent system for cardiac arrhythmia classification with Fuzzy K-Nearest Neighbors and neural networks combined with a fuzzy system", Expert Systems with Applications, vol.39, issue 3, pp.2947-2955, 2012.

[8] Maglaveras N., Stamkopolous T. etc, "ECG pattern recognition and classification using nonlinear transformations and neural networks:A review", Medical Informatics, vol.52, issue 1-3, pp.191-208, 1998.
[9] Wen Y., He L. And Shi P., "Face recognition using difference vector plus KPCA", Digital Signal Processing, vol.22, issue 1, pp.140-146, 2012.

[10] Widjaja D., Varon C.etc, "Application of Kernel Principal Component Analysis for Single-Lead-ECG-Derived Respiration", IEEE Transactions on Biomedical Engineering, vol.59, no.4, pp.1169-1176, 2012.

[11] Shalbaf A., Alizadefsani Z. And Behram H. "Echocardiography without electrocardiagram using nonlinear dimensionality reduction methods", J.Med Ultrasonics, vol.42, pp.137-149, 2015.

[12] Perry T., Zha H. etc, "Supervised Laplacian Eigenmaps with Applications in Clinical Diagnostics for Pediatric Cardiology", Computer Science \& Learning, 2012.

[13] Vozda M., Cerny M., "Methods for derivation of orthogonal leads from 12-lead electrocardiogram:A review", Biomedical Signal Processing and Control, vol.19, pp.23-34, 2015.

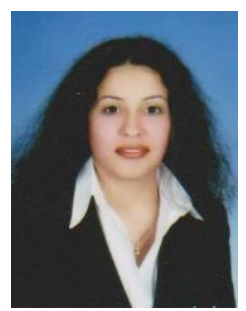

\section{BIOGRAPHY}

CIGDEM BAKIR was born in İstanbul. She received the B.S. degrees in computer engineering from the University of Sakarya, in 2010 and the M.S. degree in computer engineering from Yildiz Technical University, Istanbul, in 2014.

Since 2012, she was a Research Assistant with the Yildiz Technical University. Her research interests include recommendation systems, data mining, image processing and biomedical signal processing. 